\title{
Deep-inelastic scattering on intrinsic charm quarks in the proton
}

\author{
G. Ingelman \\ Deutsches Elektronen-Synchrotron DESY, Notkestrasse 85, D-2000 Hamburg 52 \\ and Department of Radiation Sciences, Uppsala University, Box 535, S-751 21 Uppsala, Sweden \\ L. Jönsson and M. Nyberg \\ Department of Physics, Lund University, Sölvegatan 14, S-223 62 Lund, Sweden
}

(Received 21 December 1992)

\begin{abstract}
Possibilities of probing the predicted intrinsic charm quark component in the proton by deep-inelastic lepton scattering are investigated. Total cross sections are found to be sizable, but the observable rate, e.g., through muons from semileptonic charm decays, depends strongly on the experimental situation. The DESY ep collider HERA has acceptance losses close to the proton beam, along which the ep system is strongly boosted, whereas for fixed target muon scattering, e.g., at Fermilab, the acceptance can be made much better. Backgrounds are calculated and ways to suppress them developed, resulting in acceptable signal/background ratios. For existing experiments the predicted statistics are relatively small, but may still contribute to a solution of the open intrinsic charm problem, whereas a dedicated experiment should settle the issue.
\end{abstract}

PACS number(s): $13.60 . \mathrm{Hb}, 12.38 . \mathrm{Qk}, 14.20 . \mathrm{Dh}$

\section{INTRODUCTION}

The charm production cross section in hadron collisions has been observed [1] to be larger and have a flatter distribution in Feynman $x$ than anticipated based on leading-order perturbative QCD diagrams and simple hadronization models. As a solution to this apparent problem the hypothesis of intrinsic charm (IC) was introduced [2] by assuming the existence of a $c \bar{c}$ pair as a nonperturbative component in the bound-state proton. This means that the Fock-state decomposition of the wave function, $|p\rangle=\alpha|u u d\rangle+\beta|u u d c \bar{c}\rangle+\cdots$, contains a small, but finite, probability $\beta^{2}$ for such an intrinsic quark-antiquark pair. Viewed in an infinite momentum frame, all nonperturbative (long-lived) components must move with essentially the same velocity in order that the proton can "stay together" for an appreciable time. The larger mass of the charmed quarks then implies that they take a larger fraction of the proton momentum. For definiteness we shall assume the model form for the intrinsic charm quark density distribution proposed in Ref. [2]

$$
c(x)=18 x^{2}\left\{\frac{1}{3}(1-x)\left(1+10 x+x^{2}\right)+2 x(1+x) \ln x\right\},
$$

having a mean value $\bar{x}=2 / 7$ as compared to $\bar{x}=1 / 7$ for the light quark distribution in the $|u u d c \bar{c}\rangle$ state. This distribution is derived by assuming a five-quark Fock state wave function which varies inversely with the invariant mass of the intermediate state. The normalization factor is chosen to correspond to $1 \%$ probability for intrinsic charm as first suggested [2] to explain the original data [1]. The actual normalization of heavy quark Fock components in the proton is the key unknown, although it should decrease as $1 / m_{Q}^{2}$.
More recent calculations have shown that the next-toleading-order QCD corrections to the conventional perturbative mechanism for hadroproduction of open charm give a sizable increase of the cross section (although the shapes of some important differential distributions are not much affected), see [3] and references therein. In addition, the $x_{F}$ spectra become harder than predicted by lowest-order fusion processes when string hadronization effects and coalescence with spectator quarks are taken into account $[4,5]$. This gives less of a need for an intrinsic charm component to describe the total charm production cross section, but some combination of coalescence and intrinsic charm contributions seems to be needed in order to describe the $x_{F}$ and nuclear dependence of the charm data [5]. It is also interesting to note that measured cross sections for $J / \psi$ production at large $x_{F}$ in $\pi N$ and $p N$ collisions [6] appear to be in excess of that predicted by the conventional fusion subprocesses; an effect which cannot be accounted for by string hadronization or coalescence effects. Further data [7] shows that the $J / \psi$ produced in $\pi^{-} N$ collisions becomes strongly longitudinally polarized at large $x_{F}$, suggesting a change in the production mechanism as the quarkonium state receives a high fraction of the beam momentum. The presence of an intrinsic charm contribution can account for the measured $x_{F}$ distribution as well as the observed diffractivelike nuclear target dependence of the hidden charm cross sections $[8,9]$.

The "intrinsic" quark sea should be contrasted against the "extrinsic" sea generated by large momentumtransfer processes in the perturbative QCD evolution of the parton distribution functions. Being perturbative, the latter is a short-lived fluctuation which can only be put on-shell by a large momentum-transfer external probe. The extrinsic quarks will only carry small momentum fractions of the proton as is characteristic for normal sea 
quarks. This is a normal leading-twist contribution as opposed to the higher-twist nature of the intrinsic heavy quark component. Since the intrinsic contributions are associated with multiparton correlations in the hadron wave function, the intrinsic charm quarks may carry a large fraction of the proton's momentum.

Both of these charm quark components in the proton can be probed by deep-inelastic scattering (DIS). The dominant charm production process is the photon-gluon fusion (PGF) process $\gamma g \rightarrow c \bar{c}$. Data from the European Muon Collaboration on $F_{2}^{\text {charm }}(x)=2 e_{c}^{2} x c(x)$ were found to be globally well described by this conventional process, but could not exclude an IC component below $0.6 \%$ [10]. A refined theoretical analysis [11], which took proper account of the reduction of $c(x)$ at large $x$ due to the charm threshold at low $Q^{2}$ and the QCD evolution, showed no conflict between the data and the IC model. On the contrary, it gave some evidence for an intrinsic charm component. At $x \gtrsim 0.25$, where PGF is expected to drop fast and IC should give its main contribution, the data seem to indicate the presence of IC at the level of approximately $0.3 \%$ [11].

In the following we investigate to what extent this rather unclear situation can be improved by observing deep-inelastic scattering on intrinsic charm quarks in the proton using experiments at higher energies. In particular we make detailed calculations, based on Monte Carlo event simulation, for fixed target scattering at Fermilab energies and $e p$ collisions at the DESY HERA. Charm can favorably be tagged through its semileptonic decay into muons. The branching ratio of approximately $10 \%$ is large compared to most other charm branching ratios. Furthermore, the efficiency for detecting and identifying muons is high. The reconstruction of specific charm states requires a completely different experimental situation with excellent particle identification and momentum measurement as well as good photon detection. Even so the reconstruction efficiency turns out to be low due to the large number of charm decay channels, all with small branching ratios, and due to the high combinatorial background.

As a potential background to the charm muon signal we consider, in addition to the PGF process, also muons from $\pi$ and $K$ decays, since their large production cross section in normal DIS can outbalance the small decay probability within the detector volume. Muons can also be produced in pairs from various QED radiation processes [12]. These have, however, either a small cross section or involve small momentum transfers and will then have different topologies compared to the IC signal. We have, therefore, not considered these backgrounds in detail.

\section{MODELS FOR SIMULATING SIGNAL AND BACKGROUNDS}

The basic scattering on an intrinsic charm quark is identical to normal DIS, when quark masses can be neglected, with the kinematic variables $Q^{2} \equiv-q^{2}$ $=-\left(p_{e}-p_{e^{\prime}}\right)^{2}, x \equiv Q^{2} / 2 P \cdot q$ and $y \equiv P \cdot q / P \cdot p_{e}$ having their normal definition and meaning. Furthermore, the electroweak cross sections are obtained from the normal formulas [13] by simply keeping only a charm quark distribution which is now taken from Eq. (1). It is only meaningful to consider neutral current interactions, since the charged current exchange has a smaller cross section and turns the charm quark into a lighter flavor which gives a less clear signature. The cross section for $e p$ or $\mu p$ scattering on charm is then given by

$$
\frac{d^{2} \sigma}{d x d Q^{2}}=\frac{2 \pi \alpha^{2}}{x Q^{4}}\left[1+(1-y)^{2}\right] F_{2}^{c}\left(x, Q^{2}\right),
$$

where in the case of intrinsic charm

$$
F_{2}^{c}\left(x, Q^{2}\right)=F_{2}^{\mathrm{IC}}\left(x, Q^{2}\right)=2 e_{c}^{2} x c\left(x, Q^{2}\right)
$$

and only photon exchange is taken into account since one can neglect the region of large $Q^{2}$ where $Z$ exchange contributes.

We have adopted the ep scattering event generator LEPTO 5.2 [14] to simulate complete intrinsic charm scattering events. When implementing the intrinsic charm quark density distribution equation (1) we have kept the $1 \%$ normalization; rescaling to a different value can be trivially made in our final results. We take the $Q^{2}$ evolution of $x c(x)$ into account through the leading logarithm QCD, since it has the important effect to reduce the function in the characteristic large- $x$ region as shown in Fig. 1(a). The proper evolution equation, including the charm quark mass, is rather involved and we have adopted a suitable parametrization of the numerical results in Ref. [11] by a polynomial with an amplitude proportional to $\ln \ln Q^{2}$. We normally require $Q^{2} \geq 10 \mathrm{GeV}^{2}$ to avoid corrections for mass (threshold) effects in Eq. (1) [11]. This minimum $Q^{2}$ also ensures a proper DIS situation where the scattered lepton can be detected and used to
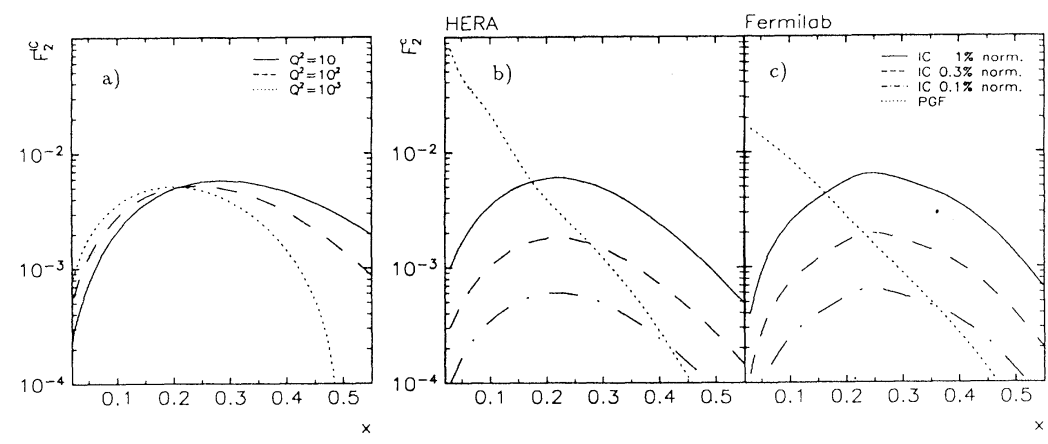

FIG. 1. (a) The intrinsic charm structure function $F_{2}^{\mathrm{IC}}\left(x, Q^{2}\right)=\frac{8}{9} x c\left(x, Q^{2}\right) \quad$ obtained from Eq. (1) taking QCD $Q^{2}$ evolution into account based on Ref. [11]. (b) and (c) The charm structure function $F_{2}^{c}$ extracted using Eq. (2) from the differential cross section obtained from the model simulations of intrinsic charm in the proton (with the indicated normalizations) and photon-gluon fusion into $c \bar{c}$ at (b) HERA and (c) Fermilab energies. $Q^{2}$ is integrated from $10 \mathrm{GeV}^{2}$ to the kinematic limit (its mean value is smaller for IC). 
reconstruct the kinematics of the event.

Although there are theoretical arguments for the form of the intrinsic charm distribution in Eq. (1) it is not necessarily fully correct. In order to investigate the sensitivity to variations in the $x$ shape we have switched off the QCD evolution, thus making the distribution somewhat harder, and found that our final results, in the form of cross sections, decrease about $5 \%$ (for Fermilab) to $10 \%$ (for HERA). These small changes do not alter the conclusions of our study.

In order to have a proper description of the hadronic final state we include QCD corrections by standard initial- and final-state parton showers [14]. A primordial transverse momentum of order $m_{c}$ is introduced through a Gaussian distribution with a width of $1 \mathrm{GeV}$. Hadronization is finally performed using the Lund string model Monte Carlo JETSET 6.3 [15]. Here, a complication arises since the scattered charm quark (antiquark) leaves a more complex proton remnant which, in addition to the three valence quarks, contains the "partner" charm antiquark (quark) to conserve the charm quantum number, cf. Fig. 2(a). Following the general strategy in LEPTO we let this partner pick up a random quark (diquark) to form a charm meson (baryon), taking, from the complete spectator system, an energy-momentum fraction chosen according to the characteristically hard charm fragmentation function of Peterson et al. [16], which is related theoretically to the intrinsic charm quark distribution. The remaining spectator diquark (quark) will then be connected to the scattered charm quark (antiquark) with the Lund string via any gluons emitted in the parton shower (Fig. 2). The details of this modeling of the target remnant is not important for our purposes, since it only affects the leading particles in the "spectator jet" which are lost in the beam pipe in an $e p$ collider and are very soft in a fixed target configuration.

The PGF background is simulated using AROMA 1.2 [17], which is based on the exact matrix elements for $\gamma g \rightarrow c \bar{c}$ in leading-order QCD [18]. The next-to-leadingorder corrections to this process have been available for some time for the photoproduction case $\left(Q^{2} \approx 0\right)[19]$ and have very recently been calculated also for DIS [20,21], which is of direct interest in our case. These corrections increase the cross section by 50-100\% [21]. Although there are some changes in the shapes of differential distributions, one may as a first approximation regard the corrections as an overall normalization change. These order $\alpha^{2} \alpha_{s}^{2}$ cross sections are not implemented in general purpose Monte Carlo generators, giving complete final
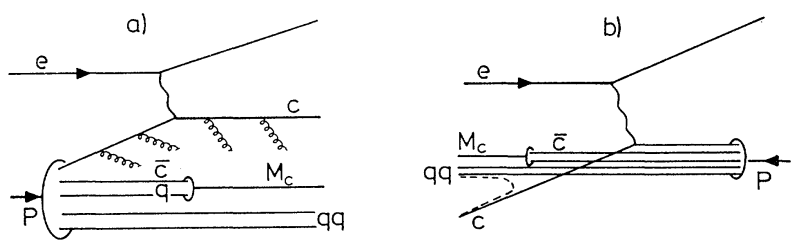

FIG. 2. Deep-inelastic scattering on an intrinsic charm quark illustrating (a) the simulation model and (b) the typical event topology in the HERA laboratory frame. states which are needed for our study of the experimental measurement of charm production. Our results are therefore based on the leading-order formalism, which should provide a good approximation with respect to the shapes of differential distributions, but the overall normalization may need to be adjusted by a simple rescaling. The influence of higher-order corrections on the event properties are, however, included in AROMA through multiple parton emission in terms of parton shower development from the $c \bar{c}$ state. To get a complete final state of observable hadrons the Lund string model for hadronization is applied in its Monte Carlo form [15]. To check the rate of muons from decays of noncharm particles we simulate normal DIS events using LEPTO 6.1 [14] and JETSET 7.3 [15] and allow particles to decay within the volume of the detector under consideration.

\section{III. ep COLLISIONS AT HERA}

In the HERA collider at DESY, 30-GeV electrons collide with 820-GeV protons giving a c.m. system (c.m.s.) energy $\sqrt{s}=314 \mathrm{GeV}$. The invariant mass $W$ of the hadronic final state is normally far above the charm production threshold $\left[W^{2}=Q^{2}(1-x) / x+m_{p}^{2} \gg \sim 4 m_{c}^{2}\right]$ and also $Q^{2}$ can easily be chosen large enough that charm quark mass effects can be safely neglected (cf. Sec. II and [11]). The two general purpose experiments, $\mathrm{H} 1$ and ZEUS, cover essentially the whole solid angle except for $\sim 4^{\circ}$ around the beam pipes. Data taking has started this year and should later give data samples of $100 \mathrm{pb}^{-1} /$ year with the design luminosity. In this section we present results obtained from the application of our simulation models for the IC signal and the background processes to the case of $e p$ scattering at HERA.

An overview can be obtained in terms of the effective charm structure function $F_{2}^{c}$, shown in Fig. $1(\mathrm{~b})$, which is extracted from Eq. (2) where the differential cross section has been generated using our model simulations for IC and PGF, respectively (accounting for mass threshold effects). As can be seen, the IC contribution dominates over PGF at large $x$, but the crossover point depends on the overall normalization of the IC component. (The mean $Q^{2}$ in an $x$ bin is, however, somewhat lower for IC than for PGF and will therefore give a correspondingly larger cross section when the $1 / Q^{4}$ factor from Eq. (2) is applied to $F_{2}^{2}$.) In the following we investigate the properties of IC events and how an enriched sample of them can be obtained.

\section{A. Event topology}

The event topology at HERA is strongly influenced by the large boost of the ep c.m.s. system in the direction of the proton beam. Large momentum fractions $x$ of the incoming quark imply that it is scattered to a small angle (unless $Q^{2}$ is very large, which is suppressed in the neutral current (NC) cross section). This event topology, which is characteristic for scattering on intrinsic charm quarks, is illustrated in Fig. 2(b). Based on our simulation we show in Fig. 3 representative rapidity distributions for those bins in $x$ and $Q^{2}$ where the IC contribution is relatively large. At the parton level (top row) we 

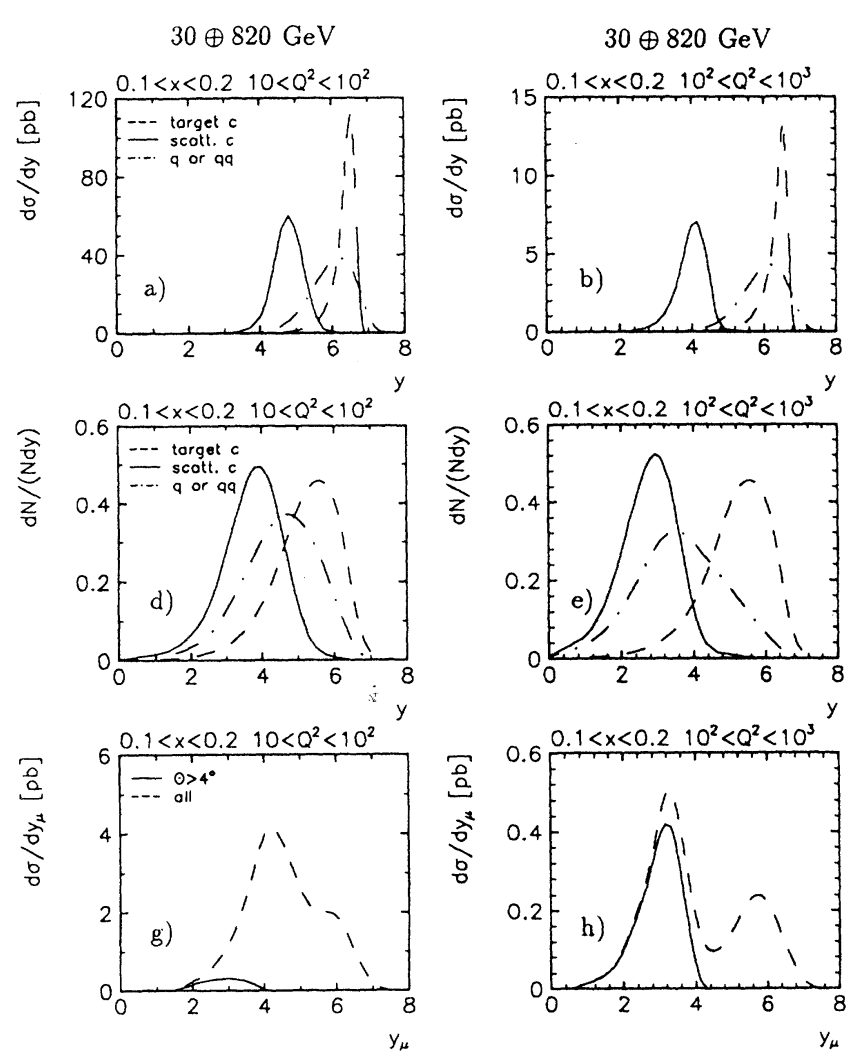

notice that the charm hadron formed from the proton remnant (target $c$ ) is emitted at large forward rapidities (i.e., along the proton beam) as is also the case for the remaining proton fragment ( $q$ or $q q$ ) although this has a somewhat broader rapidity distribution. The scattered IC quark emerges at relatively smaller, but still very much forward, rapidities. With increasing $Q^{2}$ [Fig. 3(b)] or reduced proton energy [Fig. 3(c)] it moves towards lower rapidities. After parton showers and hadronization the same picture holds (middle row in Fig. 3) although the distributions are shifted down in rapidity. With a beam pipe cut of $\theta \geq 4^{\circ}$, which corresponds to pseudorapidity $\eta \leq 3.4$, it is obvious that there will be large acceptance losses not only of the proton remnant jet (as usual) but also of the current quark jet. This is also the case for the muons from semileptonic charm decays as shown in the bottom row of Fig. 3. It is evident that the basic scattering kinematics for IC at an ep collider is not completely compatible with the possible acceptance coverage of an experiment. This is especially true at low $Q^{2}$ and for $x \geq 0.2$ where the cross section is largest.

A way to reduce the inconveniences caused by the boost would simply be to reduce the proton-beam energy. This is evident from the example of $E_{p}=500 \mathrm{GeV}$ (on $E_{e}=30 \mathrm{GeV}$ ) shown in the rightmost column in Fig. 3 and to be compared with the same $x, Q^{2}$ bin in the leftmost one. The total cross section for IC is decreased by just a few percent, but particle distributions are shifted to lower rapidities such that the observable cross sections are actually increased. The gain in the detectable muon rate is clearly seen by comparing Figs. 3(g) and 3(i).
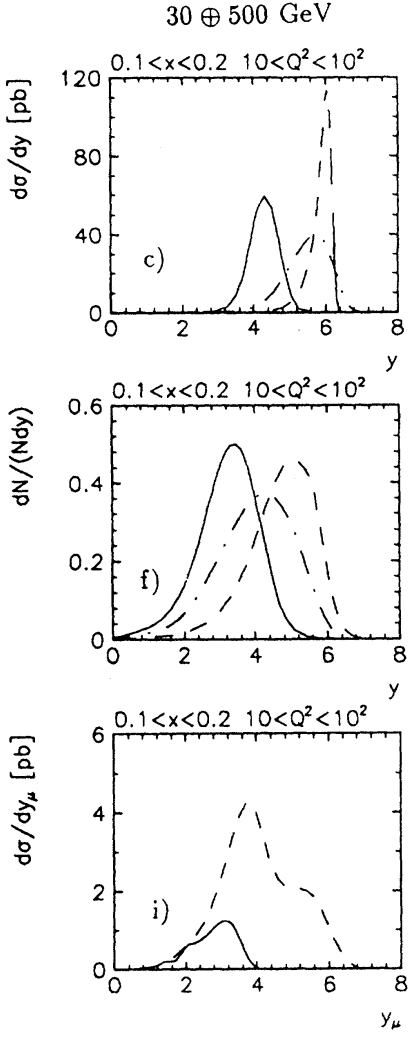

The observability of the hadronic final state varies with $x$ and $Q^{2}$, as found from the reconstruction of jets using the JADE algorithm on all particles outside the beam pipe [22]. At low $Q^{2}$ most of the hadrons are escaping down the beam pipe which makes a reliable jet reconstruction difficult. As $Q^{2}$ increases or $x$ decreases the current jet will move into the detector volume and two-jet events become predominant where the second jet often originates from the target remnant [cf. Figs. 3(d) and 3(e)].

Although the PGF background events normally have another topology with two charm jets plus the spectator, the forward boost also affects these events such that one charm jet may be lost in the beam pipe making these events more like IC events. Normal DIS events with muons from $\pi$ and $K$ decays have the same overall topology as IC, but may differ in the details.

We have here only considered muons to tag charm. Electrons and positrons from semileptonic charm decays would have the same distributions and could in principle also be used, but they are harder to identify when they are not well isolated from hadrons. There are also more background $e^{ \pm}$from various other particle decays.

\section{B. Cross sections and signal-to-background ratios}

Table I gives the cross sections for both intrinsic charm and the background processes in relevant $x, Q^{2}$ bins, with the ones we consider most promising for an IC search based on muons emphasized in boldface. Compared to the PGF background (obtained from leadingorder QCD as discussed above), which is expected to give 
TABLE I. Cross sections.

\begin{tabular}{|c|c|c|c|c|c|c|}
\hline$x$ & $10^{1}-10^{2}$ & $\begin{array}{c}\sigma_{\text {tot }}(\mathrm{pb}) \\
Q^{2}\left(\mathrm{GeV}^{2}\right. \\
10^{2}-10^{3}\end{array}$ & $10^{3}-10^{5}$ & $10^{1}-10^{2}$ & $\begin{array}{c}4^{\circ}, E_{\mu}>4 \\
Q^{2}(\mathrm{GeV} \\
10^{2}-10^{3}\end{array}$ & $10^{3}-10^{5}$ \\
\hline \multicolumn{7}{|c|}{ IC cross sections } \\
\hline $0.05-0.1$ & 24.0 & 3.40 & 0.33 & 0.72 & 0.24 & 0.02 \\
\hline $0.1-0.2$ & 59.0 & 6.60 & 0.67 & 0.40 & 0.49 & 0.05 \\
\hline $0.2-0.5$ & 75.0 & 8.10 & 0.57 & 0.03 & 0.24 & 0.05 \\
\hline $0.5-1.0$ & 1.3 & 0.29 & 0.00 & 0.00 & 0.00 & 0.00 \\
\hline \multicolumn{7}{|c|}{ PGF $c \bar{c}$ cross sections } \\
\hline $0.05-0.1$ & 170.0 & 50.0 & 4.50 & 8.02 & 4.90 & 0.38 \\
\hline $0.1-0.2$ & 49.0 & 19.0 & 2.00 & 0.40 & 1.40 & 0.21 \\
\hline $0.2-0.5$ & 5.9 & 3.3 & 0.43 & $\mathbf{0 . 0 0}$ & 0.12 & 0.04 \\
\hline $0.5-1.0$ & 0.0 & 0.0 & 0.00 & 0.00 & 0.00 & 0.00 \\
\hline \multicolumn{7}{|c|}{ DIS cross sections ( $\mu$ from $\pi, K$ decays) } \\
\hline $0.05-0.1$ & 6300 & 660 & 55.0 & 26.00 & 12.00 & 0.90 \\
\hline $0.1-0.2$ & 5700 & 560 & 53.0 & 5.60 & 8.30 & 1.20 \\
\hline $0.2-0.5$ & 3800 & 430 & 41.0 & 0.15 & 2.10 & 1.00 \\
\hline $0.5-1.0$ & 80 & 40 & 3.4 & 0.00 & 0.01 & 0.03 \\
\hline \multicolumn{7}{|c|}{ IC cross sections $(30 \oplus 500 \mathrm{GeV})$} \\
\hline $0.05-0.1$ & 24.0 & 3.3 & 0.26 & 1.10 & 0.18 & 0.01 \\
\hline $0.1-0.2$ & 59.0 & 6.6 & 0.59 & $\mathbf{1 . 3 0}$ & 0.49 & 0.04 \\
\hline $0.2-0.5$ & 75.0 & 8.8 & 0.53 & 0.47 & 0.57 & 0.04 \\
\hline $0.5-1.0$ & 1.3 & 0.3 & 0.00 & 0.00 & 0.00 & 0.00 \\
\hline \multicolumn{7}{|c|}{ PGF $c \bar{c}$ cross sections $(30 \oplus 500 \mathrm{GeV})$} \\
\hline $0.05-0.1$ & 180.0 & 46.0 & 3.50 & 8.70 & 3.30 & 0.22 \\
\hline $0.1-0.2$ & 49.0 & 17.0 & 1.70 & 1.40 & 1.60 & 0.16 \\
\hline $0.2-0.5$ & 5.7 & 3.2 & 0.38 & $\mathbf{0 . 0 3}$ & 0.27 & 0.03 \\
\hline $0.5-1.0$ & 0.0 & 0.0 & 0.00 & 0.00 & 0.00 & 0.00 \\
\hline
\end{tabular}

a larger overall cross section but decrease faster with $x$ due to the softness of the gluon distribution, we find that the IC cross section is smaller for $x \leq 0.1$, but of similar magnitude as PGF for $0.1 \leq x \leq 0.2$ and then substantially larger at higher $x$. For $x \geq 0.5$ or $Q^{2} \geq 10^{3} \mathrm{GeV}^{2}$ the absolute IC cross section is too small to be measurable with the expected HERA luminosity, and we are therefore left with the region $0.1 \leq x \leq 0.5,10^{1} \leq Q^{2} \leq 10^{3}$ for further investigation. Some differential cross sections for IC in this region are shown in Fig. 4. The characteristic hard $x$ distribution and decrease with $Q^{2}$ (photon propagator) are strongly shifted to lower $x$ and higher $Q^{2}$ when including the requirement of having both the scattered electron and charm quark within the detector $\left(4^{\circ} \leq \theta \leq 176^{\circ}\right)$, in which case they have sizable energies and transverse momenta, which favors their detection [Figs. 4(c) and 4(d)]. The latter distribution reflects the $Q^{2}$ dependence since with naive parton level kinematics the quark and electron have balancing transverse momenta given by $p_{\perp}=\sqrt{Q^{2}(1-y)}$. Since $x \geq 0.1$ and $Q^{2} \leq 10^{3}$ in our kinematic region, $y=Q^{2} / x s$ becomes $\leq 0.1$, i.e., the electron energy is lowered by at most $10 \%$ leading to the narrow peak in the electron energy distribution Fig. 4(c). As $y$ approaches smaller values it becomes increasingly difficult to reconstruct the kinematic variables from the electron alone. However, the range in which the kinematics can be reconstructed can be extended by also using the information from the hadronic system via, e.g., the double-angle method [23].
In the favored region for IC, the cross section for charm production and observable muons in the PGF background process is of similar magnitude, compare, e.g., the boldface bins in Tables $I(a)$ and $I(b)$ (where muons are required to be within the detector acceptance
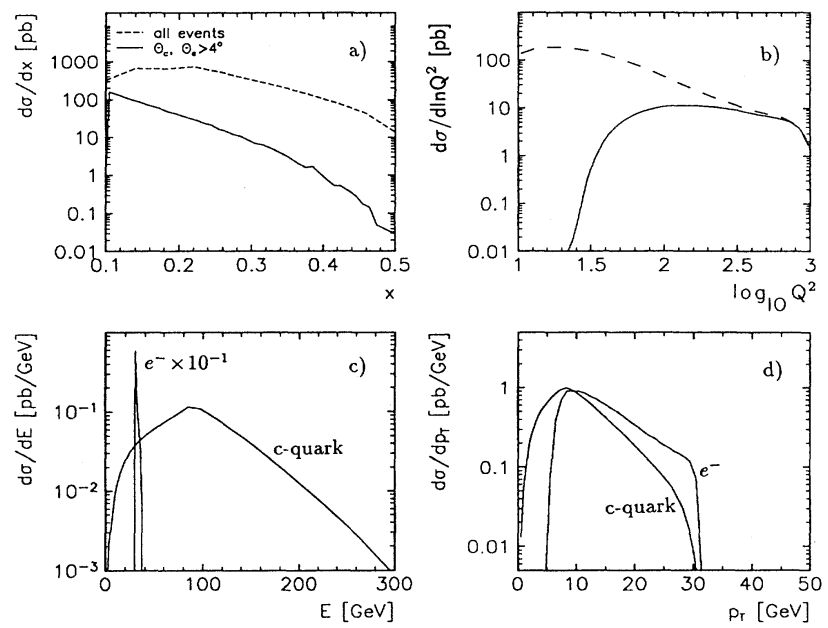

FIG. 4. Intrinsic charm cross-sections differential in (a) $x$, (b) $\log _{10} Q^{2}$ and (c) energy, (d) $p_{\perp}$ of scattered electron and quark for all events (dashed curves) and for events where both the scattered electron and the charm quark are within the detector $\left(4^{\circ} \leq \theta \leq 176^{\circ}\right)$ (full curves). 

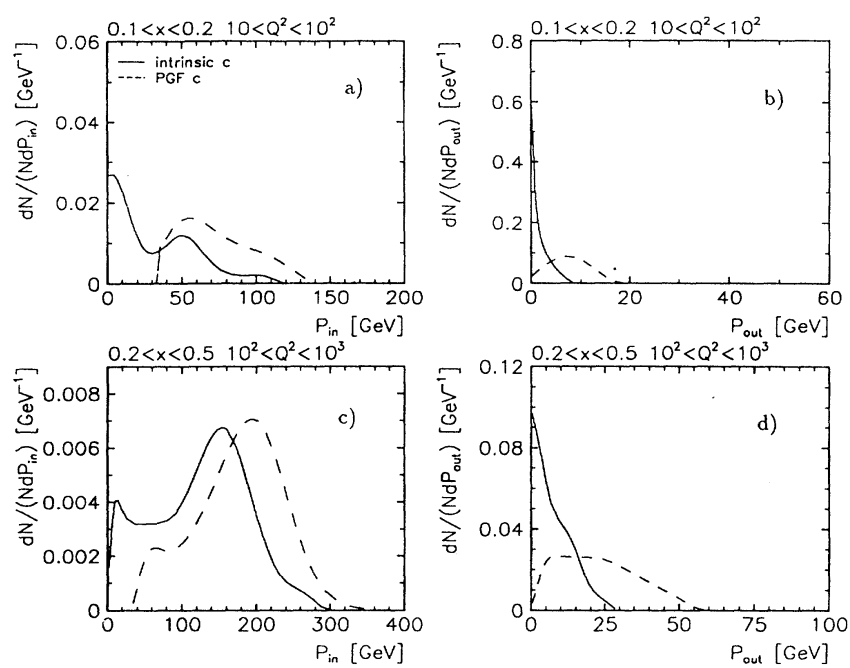

FIG. 5. Distributions of $P_{\text {in }}$ and $P_{\text {out }}$ for IC and PGF $\rightarrow c \bar{c}$ events for two of the bins in $x$ and $Q^{2}$ marked in Tables $I(a)$ and I(b).

and have energy above $4 \mathrm{GeV}$ to ensure their identification). Although this may be sufficient to prove an excess in the charm production over the conventional process, one would like to obtain a cleaner IC sample. This can be based on the expected difference in event topology as discussed above. Letting the current jet direction (taken as the reconstructed jet at the largest polar angle) and the beam axis define a plane, we consider the sum of all particle momenta in this plane $\left(P_{\text {in }}\right)$ and out of the plane $\left(P_{\text {out }}\right)$. The differences observed in these quantities, see Fig. 5, reflect the extra $c$ quark jet in PGF which gives additional transverse momenta and makes the PGF events less planar. (The peak at small $P_{\text {in }}$ in Figs. 5(a) and 5(c) is due to the partial loss of a jet in the beam pipe.) A cut, in particular in $P_{\text {out }}$, can then be applied to suppress the background without affecting the signal much, as exemplified in Table II based on the muon sample.

Muons from ordinary meson decays constitutes a problem since the low decay probability in the detector is compensated by the large cross section for $\pi$ and $K$ production in normal DIS events as indicated in Table $I(c)$. Given the softer energy and $p_{\perp}$ distribution of these muons, see Fig. 6, an increased cut in muon energy (or a cut in $p_{\perp}$ ) would improve the signal-to-background ratio, but a cut which gives an acceptable ratio would lead to a significant loss of the signal cross section. We have checked that these remaining high-energy background muons cannot be excluded based on their tracks having

TABLE II. Separation of IC and PGF.

\begin{tabular}{ccccc}
\hline \hline & $Q^{2}$ & $P_{\text {out }}$ cut & $\sigma_{\text {IC }}$ & Signal \\
\cline { 4 - 5 }$x$ & $\left(\mathrm{GeV}^{2}\right)$ & $(\mathrm{GeV})$ & $(\mathrm{pb})$ & background \\
\hline $0.1-0.2$ & $10^{1}-10^{2}$ & 7.5 & 0.38 & 3.5 \\
$0.1-0.2$ & $10^{2}-10^{3}$ & 10.0 & 0.33 & 1.0 \\
$0.2-0.5$ & $10^{2}-10^{3}$ & 20.0 & 0.24 & 4.4 \\
\hline \hline
\end{tabular}
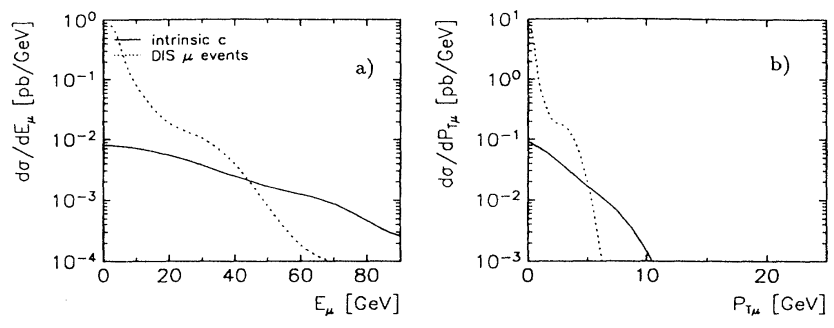

FIG. 6. Energy and transverse momentum distributions of muons in IC events (full curves) and from $\pi, K$ decays in normal DIS events (dotted curve). $0.2<x<0.5,10^{2}<Q^{2}<10^{3}$.

observable kinks or being incompatible with originating from the primary vertex. However, since the muons in IC come from the decay of a leading particle they should be more strongly correlated with the quark jet direction as compared to the majority of decay muons from $\pi, K$ which are not necessarily leading particles. We have therefore compared the distance $\Delta R=\sqrt{\left(\eta_{j}-\eta_{\mu}\right)^{2}+\left(\phi_{j}-\phi_{\mu}\right)^{2}}$ between the muons and the reconstructed current jet and indeed found such a difference, but the background suppression due to this difference alone is still not sufficient. Thus, there is no simple cut to avoid this background, but one may investigate the feasibility of neural networks, which have proven useful for classification problems based on multidimensional inputs. The alternative way is to subtract such decay muons since they come from well-known decay processes of particles whose production it should be possible to control sufficiently well based on direct measurements and, perhaps, complemented with tuned Monte Carlo event generators. Therefore, we do not consider this background to be a major problem.

\section{MUON SCATTERING ON FIXED TARGET}

From the last section it is evident that the main problem in observing DIS on charm quarks in ep collider experiments is due to the limited acceptance coverage close to the downstream proton beam pipe. This does not only apply when tagging charm through semileptonic decays into muons, but also in attempts to reconstruct charm particles through invariant masses (e.g., using $D^{*} \rightarrow D \pi$ ) since tracking and momentum measurements of highenergy particles at small forward angles close to the beam pipe are very difficult. These experimental problems can in general be reduced in fixed target experiments since a much smaller hole in the detector is needed to let through the primary lepton beam. A further advantage in a fixed target configuration is that it allows much more freedom to optimize the detector arrangement so as to obtain the best possible signal-to-background ratio. In particular, the hadron absorber can be placed close to the target in order to minimize the amount of muons from $\pi$ and $K$ decays.

In order for the cross section for IC not to be suppressed by the charm particle masses, the lepton-beam energy cannot be too low. For a beam energy of $E_{e}=30$ $\mathrm{GeV}$ (as for the HERMES experiment at HERA) the to- 
tal IC cross section is only $\sim 8 \mathrm{pb}$, so electron accelerators are practically excluded and we are left with muon beams from proton accelerators. However, once the lepton-beam energy is above a few hundred $\mathrm{GeV}$, i.e., $\sqrt{s} \gtrsim 20 \mathrm{GeV}$, the total IC cross section does not vary much with increasing energy. At Fermilab, with $E_{\mu} \simeq 470 \mathrm{GeV}(\sqrt{s}=30 \mathrm{GeV})$, the IC cross section is still $20 \%$ higher than at CERN with $E_{\mu}=280 \mathrm{GeV}(\sqrt{s}=23$ $\mathrm{GeV})$, but only $30 \%$ lower than at HERA $(\sqrt{s}=314$ $\mathrm{GeV})$. Thus, the gain in cross section at collider energies is not essential.

These overall relations of cross sections at Fermilab and HERA energies are demonstrated in Figs. 1(b) and 1 (c), which show the effective charm structure functions $F_{2}^{c}$ (see the definition and comments in the second paragraph of Sec. III). Threshold effects are included in the simulations and one can therefore make a proper comparison between the different energies. Whereas the IC cross section does not increase much in this energy range, the PGF background does increase significantly. In the latter process, the $c \bar{c}$ pair is produced in the photon-gluon subsystem which has a lower invariant mass as a result of the, typically small, fractional energy of the proton carried by the gluon. Hence, the charm mass threshold gives a stronger effect in this case, resulting in a stronger energy dependence. Comparing $F_{2}^{\mathrm{IC}}$ and $F_{2}^{\mathrm{PGF}}$ in Figs. 1 (b) and 1(c), it is evident that the signal-to-background ratio is more favorable at Fermilab energies than at HERA and that the crossover point in $x$, over which IC dominates, is favorably located at smaller $x$ values in the Fermilab case.

As far as luminosity is concerned, the many-orders-ofmagnitude larger density of protons in a liquid or pressurized gas target compared to a circulating proton beam are compensated by the lower number of muons per second impinging on the target as compared to the number of electrons in an $e p$ collider. To evaluate the attainable luminosities with the Fermilab muon beam we use parameters typical for the E665 experiment [24]: muon flux of $2 \times 10^{7}$ muons in a $20 \mathrm{~s}$ spill length with $57 \mathrm{~s}$ cycle time. The typical target thickness used was $10 \mathrm{~g} / \mathrm{cm}^{2}$ resulting in luminosities of the order $10^{30} \mathrm{~cm}^{-2} \mathrm{~s}^{-1}$. Assuming a $50 \%$ efficient running time, this would in turn give an integrated luminosity of about $15 \mathrm{pb}^{-1}$ per year. Comparing this with the design luminosity $10^{31} \mathrm{~cm}^{-2} \mathrm{~s}^{-1}$ at HERA, giving $100 \mathrm{pb}^{-1}$ per year, and considering the similar magnitude of the IC cross section, it is clear that the statistics attainable in a fixed target experiment can only be comparable to or larger than at HERA if either a denser target is used and/or the acceptance losses are correspondingly smaller. In order to clarify the acceptance situation for the fixed target case one needs to investigate the detailed properties of the IC and the background events.

\section{A. Event topology}

In a fixed target experiment the $\mu$ p c.m.s. is strongly boosted along the muon beam and hence the scattered muon and the current jet will appear at rather small forward angles. As an example, in order to give definite numerical results, we will in what follows take the case of a
470-GeV muon beam on a fixed proton target corresponding to a feasible experiment at Fermilab. The polar angles of the scattered muon and the current jet, i.e., the scattered quark using quark-parton model kinematics, is shown in Fig. 7 for the region in $x, Q^{2}$ of interest for IC. In order to determine the event kinematics, i.e., $x$ and $Q^{2}$, one needs to measure the scattered muon and therefore, according to Fig. 7 , the region $0.25^{\circ} \lesssim \theta_{\mu} \lesssim 5^{\circ}$ has to be covered. Similarly, Fig. 7 shows that the angle of the scattered quark varies between $\sim 1^{\circ}$ and $25^{\circ}$. A muon from the decay of a scattered charm quark would essentially emerge at the same angle since the charmed particle momentum is normally much larger than the transverse momenta generated in its decay.

The distribution of muons from IC decays in terms of their energy and angle is shown in Fig. 8. As expected from basic kinematics of the scattered quark, there is a correlation such that higher muon energies occur at smaller angles. Furthermore, the muon angle tends to increase and its energy decrease for larger $x$ [compare Figs. $8(a)$ and $8(b)$ ]. The scattered intrinsic charm quark (antiquark) will leave its partner charm antiquark (quark), from the intrinsic $c \bar{c}$ pair, in the target remnant. As described in Sec. II, the latter will therefore produce a charmed meson (baryon) in the target fragmentation region, i.e., at small laboratory energies but possibly much larger angles than hadrons in the current jet. Semileptonic decays of such charmed hadrons gives rise to the component of low-energy muons extending to larger angles as shown in Figs. 8(c) and 8(d). These muons provide another signature for IC and one should therefore attempt to detect them, too.

The identification of muons is normally based on their ability to penetrate large amounts of material. With the exception of neutrinos, which are not seen in the detector in any case, this is not true for other particles. The experimental technique is thus to use an absorbing material

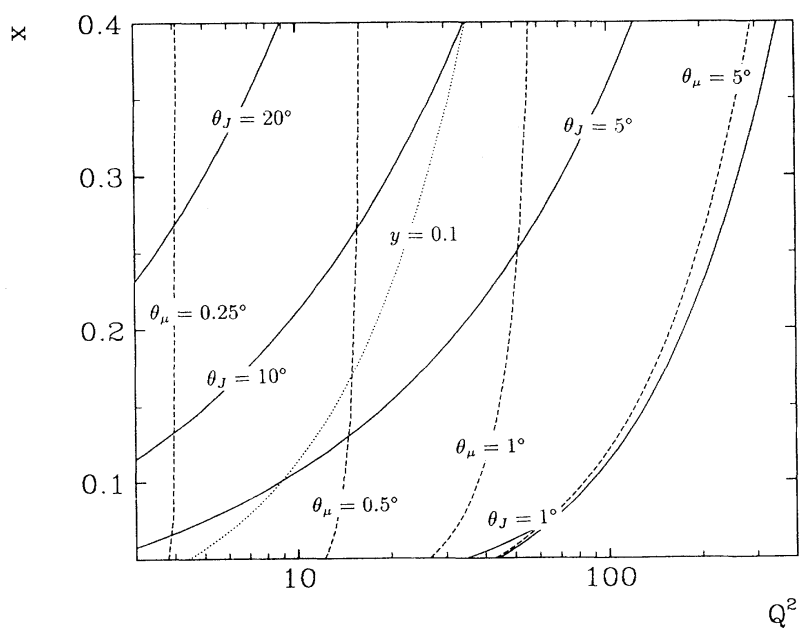

FIG. 7. Isolines for constant polar angles $\theta_{J}$ of the current jet, i.e., the scattered quark (full lines), and $\theta_{\mu}$ of the scattered muon (dashed lines) in the kinematic $x, Q^{2}$ plane for DIS of 470 $\mathrm{GeV}$ muons on a fixed nucleon target. Lines of constant $y$ are also shown. 

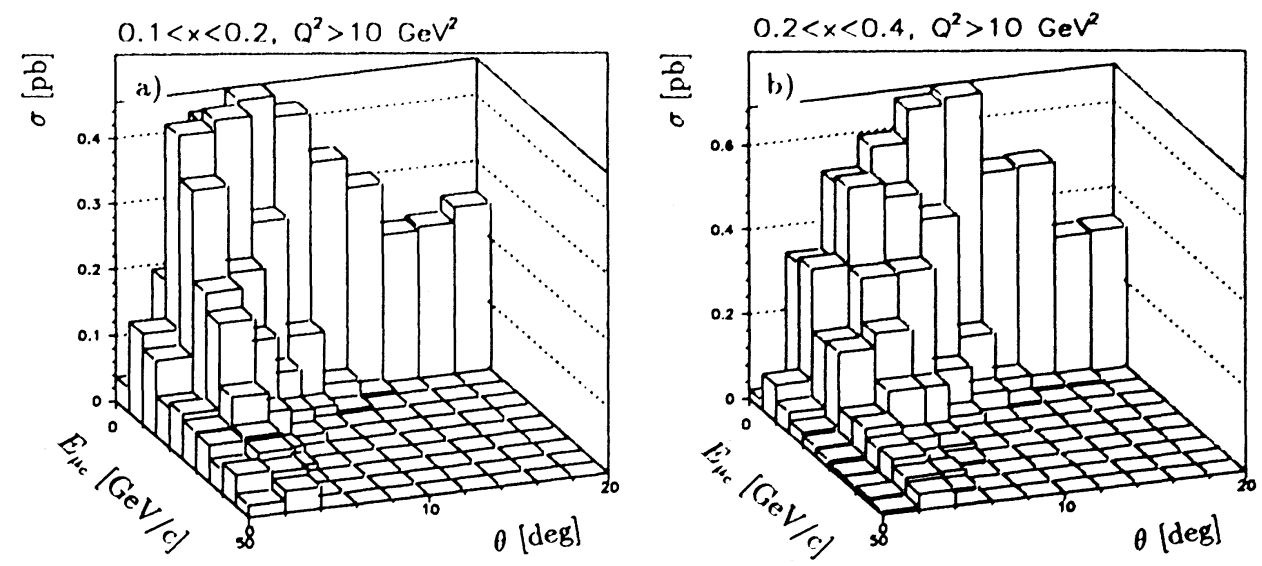

FIG. 8. Differential cross section ( $\mathrm{pb} / \mathrm{bin})$ of muons in IC events vs muon energy and polar angle for DIS of $470 \mathrm{GeV}$ muons on a fixed nucleon target. The large energy muons at smaller angles originate from the decay of the scattered intrinsic charm quark (antiquark), whereas those at lower energies and larger angles [better seen in (c) and (d)
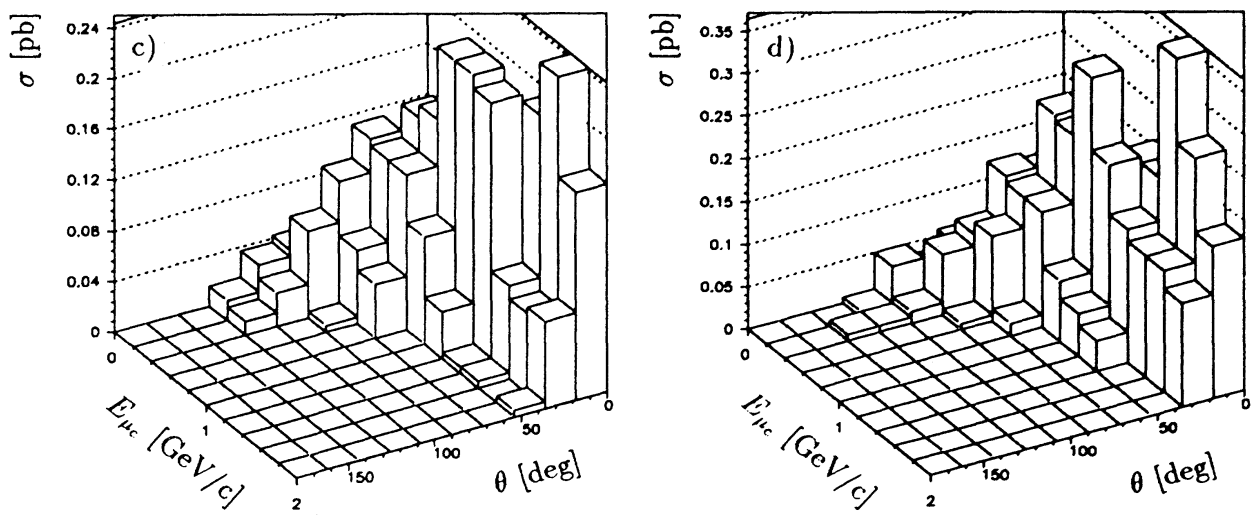
with adjusted scales] are dominantly from the decay of the partner charm antiquark (quark) left over in the target remnant.

of appropriate thickness to filter away all other particles except the muons.

\section{B. Cross sections and signal-to-background ratios}

The cross section for the IC signal and the leadingorder PGF background is given in Table III for the most relevant bins in $x$ and $Q^{2}$. For the total cross sections no experimental restrictions have been included, but for the muon cross sections we have assumed a fully efficient detector covering the polar angle region from $0.25^{\circ}$ to $10^{\circ}$ where the lower limit corresponds to $Q^{2} \sim 5 \mathrm{GeV}^{2}$ (see Fig. 7). The muons were required to have an energy of at least $10 \mathrm{GeV}$, based on our estimations of the conditions at the E665 experiment, which should allow them to pass a thick filter giving a safe absorption of the hadrons. From Fig. 8 it is clear that if this energy requirement can be somewhat relaxed a sizable gain in the muon rate will result. For example, taking a minimum muon energy of 5 $\mathrm{GeV}$ will increase the cross section by $60 \%$, which has to be balanced against the increased probability for hadrons passing through a correspondingly less thick absorber.

Although it is clear from Table III that a lower limit for $Q^{2}$ of $5 \mathrm{GeV}^{2}$ gives a substantially higher total cross section compared to $Q^{2} \geq 10 \mathrm{GeV}^{2}$, there is only a small gain in observable muons. This is due to the resulting lower $y$ values giving lower current jet energies, which in turn results in lower decay muon energies such that the $10-\mathrm{GeV}$ cut is more noticeable. Because of this minor difference in the observable cross section we can allow the lower angular limit to increase to $0.4^{\circ}$, which corresponds to $Q^{2} \sim 10 \mathrm{GeV}^{2}$ as is realized from Fig. 7. This of course will simplify the experimental situation. From Fig. 8 we notice that a cut on the muon energy at $10 \mathrm{GeV}$ will remove essentially all muons above $10^{\circ}$, which we therefore have chosen as our upper limit in the angle.

When comparing with the corresponding results at an ep collider (Table I) one should note that although the larger c.m.s. energy gives a larger total IC cross section, the observable muon cross section is smaller due to the re-

TABLE III. Cross sections.

\begin{tabular}{|c|c|c|c|c|c|c|}
\hline \multirow[b]{2}{*}{$x$} & \multicolumn{3}{|c|}{$\begin{array}{c}\sigma_{\mathrm{tot}}(\mathrm{pb}) \\
Q^{2}\left(\mathrm{GeV}^{2}\right)\end{array}$} & \multicolumn{3}{|c|}{$\begin{array}{c}\sigma_{\mu}(\mathrm{pb}) \\
Q^{2}\left(\mathrm{GeV}^{2}\right)\end{array}$} \\
\hline & $5-10$ & $10-45$ & $45-$ & $5-10$ & $10-45$ & $45-$ \\
\hline \multicolumn{7}{|c|}{ IC cross sections } \\
\hline $0.05-0.1$ & 21.0 & 15.0 & 1.0 & 0.60 & 0.99 & 0.08 \\
\hline $0.1-0.2$ & 49.0 & 43.0 & 6.0 & 0.47 & 1.70 & 0.50 \\
\hline $0.2-0.4$ & 6.1 & 54.0 & 11.0 & 0.02 & 1.60 & 0.81 \\
\hline $0.4-0.6$ & & 3.9 & 2.7 & & 0.08 & 0.16 \\
\hline \multicolumn{7}{|c|}{ PGF $c \bar{c}$ cross sections } \\
\hline $0.05-0.1$ & 36.0 & 89.0 & 8.2 & 1.30 & 8.50 & 1.10 \\
\hline $0.1-0.2$ & 4.9 & 26.0 & 9.5 & 0.05 & 1.70 & 1.20 \\
\hline $0.2-0.4$ & 0.1 & 2.9 & 2.7 & 0.00 & 0.10 & 0.29 \\
\hline $0.4-0.6$ & & 0.0 & 0.1 & & 0.00 & 0.01 \\
\hline
\end{tabular}


duced acceptance along the downstream proton beam pipe. In the favored $x, Q^{2}$ regions the cross section at the fixed target can be a factor $\sim 4$ larger, with a muon energy cut at $10 \mathrm{GeV}$, which does not quite outbalance the lower luminosity when using a low-density target.

In order to illustrate the influence of the muon background from decaying pions and kaons, we have in Fig. 9 plotted the resulting signal/background ratio as a function of the decay distance available before the hadron absorber. To suppress this background the hadron filter should obviously be placed as close as possible to the target.

\section{A dedicated intrinsic charm experiment}

With the luminosities discussed above the rate of observable IC events, when tagged through decay muons, are about the same at the fixed target and the collider configuration. However, the prospects for improvements seem better in the fixed target case. The muon-scattering experiment E665 [24] at Fermilab, with 470-GeV muons on a liquid or pressurized gas target, is unfortunately not very well designed for IC measurements. Since the experimental program involved a study of the produced hadronic system in a spectrometer, the hadron filter and muon detector was forced to be placed far downstream $(26 \mathrm{~m})$ of the target. This not only gives a large muon background from $\pi$ and $K$ decays, but also limits the detection of high-energy muons to within $\sim 3^{\circ}$. This is no problem for the high-energy scattered muon, but for muons from charm decays it results in a substantial acceptance loss as can be seen from Fig. 8. For intrinsic charm we are only interested in detecting and measuring muons and do not care about the hadrons produced. Thus, the muon detector can be placed close to the target to increase the acceptance. Furthermore, a more dense target could preferably be chosen to increase the luminosity. By using iron the luminosity could easily be increased by one to two orders of magnitude without running the risk of absorbing the muons of interest, but one has to consider the increased radiative corrections. With such an arrangement the target would also serve as a first hadron filter.

The most energetic hadrons determine the thickness of the filter, but since in the IC process the muons are produced over a broad momentum range, one has to split up the filter into a sandwich structure where absorbing plates are interleaved with detector planes in order not to absorb the lower momentum muons before they can be identified. The muons from decays of the scattered charm quark will have a similar or higher energy compared to the hadrons in the current jet and should therefore be possible to identify. Also the low-energy muons at larger angles, arising from charm in the target remnant, should in principle be detectable since hadrons at those angles should also come from the target remnant hadronization and therefore have similar or even lower energies.

The requirements for a dedicated intrinsic charm experiment, at beam conditions such as at Fermilab, are set by the following criteria.
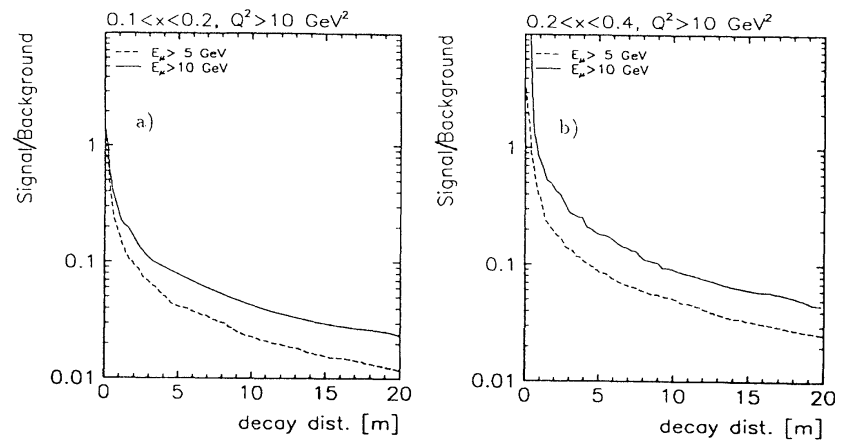

FIG. 9. Ratio of muons from the decay of scattered intrinsic charm quarks and from $\pi$ and $K$ decays in normal DIS events vs the available decay distance before the hadron absorber in the experiment. The muons are required to have a minimum energy of $5 \mathrm{GeV}$ (dashed curve) or $10 \mathrm{GeV}$ (full curve) as example criteria for identification in a muon detector. In the kinematic region used in (a) and (b), the signal cross section is $4.6 \mathrm{pb}$ requiring $E_{\mu}>10 \mathrm{GeV}$ and $7.5 \mathrm{pb}$ requiring $E_{\mu}>5 \mathrm{GeV}$.

(i) In the kinematic region of interest the scattered muon is emitted in the angular range $\sim 0.4^{\circ}-5^{\circ}$ and with momenta between $50 \mathrm{GeV} / c$ and $400 \mathrm{GeV} / c$. Since the scattered muon has to be momentum determined to extract the kinematics of the event a magnetic spectrometer with high bending power and good spatial resolution is needed.

(ii) The incoming muon beam has a certain width of typically $\pm 3 \mathrm{~cm}$ which implies that the spectrometer has to be placed at a distance from the target of at least $5 \mathrm{~m}$ to allow muons scattered by $0.4^{\circ}$ to exit the primary beam.

(iii) The momentum spread of the Fermilab primary muon beam has a sigma of about $60 \mathrm{GeV} / c$ at a nominal beam energy of $500 \mathrm{GeV}$. Therefore, the magnetic spectrometer must be able to distinguish the scattered muon from primary muons of comparable momenta. This can be achieved as in the E665 experiment with two dipole magnets with opposite polarities or with a toroid magnet with a central hole for the primary beam.

(iv) The decay muon from the scattered intrinsic charm quark emerges in the angular range $1^{\circ}-20^{\circ}$ and with momenta between a few and $50 \mathrm{GeV} / c$, while the muons from charm in the target remnant would be softer and have emission angles extending above $50^{\circ}$. It is, strictly speaking, not necessary to measure the momenta of these muons since they are only used to tag the presence of charm. To identify these muons over such a momentum range needs a sandwich arrangement of absorbers and detectors as described above. The detector could have one forward part covering angles up to $\sim 45^{\circ}$ and a barrel part for the angular range $\sim 45^{\circ}-90^{\circ}$ and it should be placed close to the target to minimize the background from decaying pions and kaons.

With a solid target giving one order of magnitude higher luminosity one could get more than 500 IC events per year. Since this number applies to a $1 \%$ normalization of the IC component in the proton, one could then reach the level $0.1 \%$, or lower, and thus be more sensitive 
than the present evidence based on European Muon Collaboration (EMC) data. Including the possibility to detect muons from the target remnant would add more data. It would also give the possibility to observe two muons in the same event, one from the scattered charm quark and one from the remaining charm in the target remnant, giving an even more characteristic signature for intrinsic charm.

\section{CONCLUSIONS}

We have found that the total cross section for scattering on intrinsic charm quarks are quite reasonable both at fixed target and HERA energies. However, since the cross section is dominant at large $x$ and rather small $Q^{2}$, this implies a typical event configuration at an ep collider where the scattered charm quark, and hence its decay muon, emerge at small forward angles giving large acceptance losses due to the beam pipe. Any possibility to cover smaller angles will give a significant improvement, e.g., lowering the cut $\theta \geq 4^{\circ}$ (applicable in the $\mathrm{H} 1$ detector) to $3^{\circ}$ would increase the observable muon cross section by $70 \%$. Alternatively, one can increase the observable cross section by lowering the proton-beam energy, since this would give a less forward boosted ep system. For example, $E_{p}=500 \mathrm{GeV}$ would more than double the observable muon rate.

These acceptance problems are not present in a fixed target experiment which is designed to measure muons over a large angular region. Thus, the slightly smaller total cross section, due to the smaller c.m.s. energy, is more than compensated for the observable muon cross section, which is in fact a factor $\sim 4$ larger. The absolute level of the cross section for the muon signal from intrinsic charm in the preferred range $0.1 \leq x \leq 0.5, Q^{2} \gtrsim 10 \mathrm{GeV}^{2}$ is around $1 \mathrm{pb}$ at HERA and $4 \mathrm{pb}$ at Fermilab when a $1 \%$ normalization of the intrinsic charm quark density distribution is assumed. At HERA this should give a useful event sample with an integrated luminosity of 100 $\mathrm{pb}^{-1}$ attainable in a year. Thus with statistics of, say, $500 \mathrm{pb}^{-1}$ obtainable in some years running it should be possible to probe the intrinsic charm content of the proton down to a level of $0.1 \%$, i.e., somewhat below the level indicated by the analysis [11] of the EMC data.

A possible increase of the luminosity in a future upgrade of HERA would, of course, be very useful whereas an increased energy has no effect. By combining the HERA proton beam with a possible linear electron accelerator, with beam energy $\gtrsim 250 \mathrm{GeV}$, we will face the problem of not being able to detect the scattered electron since it will emerge at very small angles. Only at quite large $Q^{2}$ this would be possible but then the IC cross section becomes too small.
In a dedicated fixed target experiment with a lowdensity target the observable event rate is similar to HERA, but it can be increased with a more dense target. With integrated luminosities of $\sim 150 \mathrm{pb}^{-1} /$ year one would get a factor of 6 more statistics compared to HERA and should be able to probe the $0.1 \%$ level of an intrinsic charm component in the proton in only one year of running.

There are two kinds of backgrounds to be considered. The first, and most serious one, is charm production through the photon-gluon fusion process. In the HERA case it is of the same magnitude as the signal in the preferred $x, Q^{2}$ range, while it is almost a factor 2 smaller in the fixed target situation. Given the different topology of these events we have demonstrated how they can be suppressed to an acceptable level. The second background is due to muons from decaying pions and kaons in normal DIS events. A fixed target experiment can be designed to minimize such a contribution by making the flight distance to the muon detector short. At HERA these muons are much more frequent than muons from charm, but they can be strongly suppressed by an energy cut and the remainder can be subtracted once the $\pi$ and $K$ momentum spectra are known sufficiently well.

We note that intrinsic beauty may also be present in the proton, but should be suppressed by the factor $m_{b}^{2} / m_{c}^{2} \approx 10$ relative to intrinsic charm. Together with an increased heavy quark production threshold, this gives a total cross section which is about $5 \%$ of the IC cross section at HERA and still lower at fixed target energies. Although a larger fraction of the muons from bottom decays may enter a HERA detector, due to an increased transverse momentum, the observable muon cross sections will be uncomfortably small.

Finally, it is interesting to note that a dedicated fixed target experiment for intrinsic charm could also be used with the muon beam replaced by a proton beam. This would open the possibility to investigate the predicted $J / \psi$ production $[8,25]$ from the intrinsic charm quark pair. The basic idea is here that the $c \bar{c}$ pair has a smaller transverse extension $\left(\sim 1 / m_{c}\right)$ than the light quarks and a target nucleus may therefore act as a "filter" that absorbs the valence quarks, leaving a forward $c \bar{c}$ pair hadronizing into a $J / \psi$ which can be detected through its decay to a muon pair at small forward angles.

\section{ACKNOWLEDGMENTS}

We are grateful to Stan Brodsky for several inspiring discussions and helpful comments and to H. Schellman for useful information on the E665 experiment. This work was supported by the Swedish Natural Science Research Council.
[1] D. Drijard et al., Phys. Lett. 81B, 250 (1979); 85B, 452 (1979); K. L. Giboni et al., ibid. 85B, 437 (1979); W. Lockman et al., ibid. 85B, 443 (1979); A. Chilingarov et al., ibid. 83B, 136 (1979).

[2] S. J. Brodsky, P. Hoyer, C. Peterson, and N. Sakai, Phys. Lett. 93B, 451 (1980); S. J. Brodsky and C. Peterson, Phys.
Rev. D 23, 2745 (1981).

[3] M. L. Mangano, P. Nason, and G. Ridolfi, Nucl. Phys. B373, 295 (1992); G. Altarelli et al., ibid. B308, 724 (1988).

[4] B. Andersson, H. U. Bengtsson, and G. Gustafson, Lund University Report No. LU-TP 83-4, 1983 (unpublished).

[5] R. Vogt, S. J. Brodsky, and P. Hoyer, Nucl. Phys. B383, 
643 (1992)

[6] NA3 Collaboration, J. Badier et al., Z. Phys. C 20, 101 (1983).

[7] C. Biino et al., Phys. Rev. Lett. 58, 2523 (1987).

[8] S. J. Brodsky, P. Hoyer, A. H. Mueller, and W.-K. Tang, Nucl. Phys. B369, 519 (1992).

[9] R. Vogt, S. J. Brodsky, and P. Hoyer, Nucl. Phys. B360, 67 (1991).

[10] J. J. Aubert et al., Phys. Lett. 110B, 73 (1982); Nucl. Phys. B213, 31 (1983).

[11] E. Hoffman and R. Moore, Z. Phys. C 20, 71 (1983).

[12] V. Ganapathi and J. Smith, Phys. Rev. D 19, 801 (1979).

[13] G. Ingelman et al., in Proceedings of the HERA Workshop, Hamburg, 1987, edited by R. D. Peccei (DESY, Hamburg, 1988), Vol. 1, p. 3.

[14] G. Ingelman, LEPTO version 5.2 (unpublished); LEPTO version 6.1, in Proceedings of "Physics at HERA" [26], Vol. 3, p. 1366.

[15] T. Sjöstrand, Comput. Phys. Commun. 39, 347 (1986); 43, 367 (1987); JETSET 7.3, program manual, Report No. CERN-TH.6488/92 (unpublished).

[16] C. Peterson et al., Phys. Rev. D 27, 105 (1983).
[17] G. Ingelman and G. A. Schuler, AROMA 1.2 (unpublished); see also AROMA 1.4, in Proceedings of "Physics at HERA" [26], Vol. 3, p. 1346; Z. Phys. C 40, 299 (1988).

[18] G. A. Schuler, Nucl. Phys. B299, 21 (1988).

[19] R. K. Ellis and P. Nason, Nucl. Phys. B312, 551 (1989); J. Smith and W. L. van Neerven, ibid. B374, 36 (1992).

[20] E. Laenen et al., Phys. Lett. B 291, 325 (1992); Nucl. Phys. B392, 162 (1993).

[21] E. Laenen et al., Nucl. Phys. B392, 229 (1993).

[22] For jet finding in ep collider events, see, e.g., P. N. Burrows, G. Ingelman, and E. Ros, Z. Phys. C 39, 257 (1988); M. Fleischer et al., in Proceedings of "Physics at HERA" [26], Vol. 1, p. 303.

[23] S. Bentvelsen et al., in Proceedings of "Physics at HERA" [26], Vol. 1, p. 23.

[24] E665 Collaboration, M. R. Adams et al., Nucl. Instrum. Methods A 291, 533 (1990).

[25] S. J. Brodsky and P. Hoyer, Phys. Rev. Lett. 63, 1566 (1989).

[26] Proceedings of "Physics at HERA," edited by W. Buchmüller and G. Ingelman (DESY, Hamburg, 1992), Vols. 1-3. 